\title{
La valorisation du rebut
}

Le recyclage commercial de la fripe en contexte de crise

Repurposing Rags. Commercial Recycling of Second-hand Clothes in Times of Crisis

\section{Sylvie Ayimpam}

\section{OpenEdition \\ Journals}

Édition électronique

URL : https://journals.openedition.org/tc/8034

DOI : $10.4000 /$ tc. 8034

ISSN : 1952-420X

\section{Éditeur}

Éditions de l'EHESS

\section{Édition imprimée}

Date de publication : 31 octobre 2016

Pagination : 264-279

ISBN : 9782713225291

ISSN : 0248-6016

Référence électronique

Sylvie Ayimpam, «La valorisation du rebut », Techniques \& Culture [En ligne], 65-66 | 2016, mis en ligne le 31 octobre 2018, consulté le 29 septembre 2022. URL : http://journals.openedition.org/tc/8034 ; DOI : https://doi.org/10.4000/tc.8034 


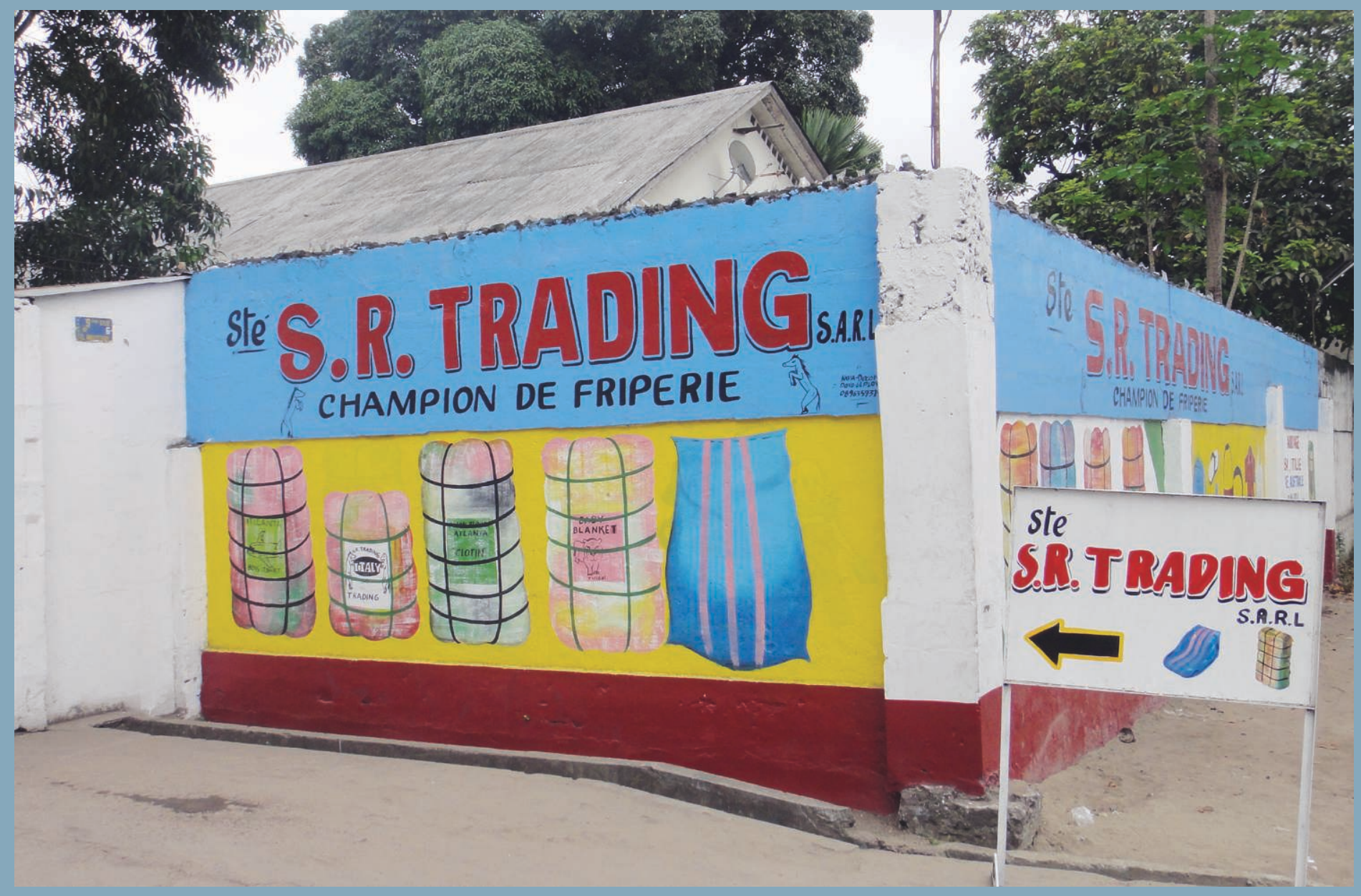




\section{La valorisation du rebut}

\section{Le recyclage commercial de la fripe en contexte de crise}

Cette contribution, consacrée à l'analyse du processus de revalorisation de la fripe dans les réseaux de vente au détail à Kinshasa, s'appuie sur l'observation des modalités concrètes et particulières de son recyclage commercial. «La fripe» désigne toutes sortes de vêtements de seconde main considérés comme rebuts dans les pays riches d'Europe et d'Amérique, qui sont exportés dans les pays Africains où ils sont recyclés commercialement. À Kinshasa, la fripe fut longtemps méprisée et considérée comme un marqueur de pauvreté. Dans cette ville où, depuis l'époque coloniale, un culte est rendu à l'élégance vestimentaire, les citadins accordaient une importance extrême à la qualité du prêt-à-porter. Mais, depuis un peu plus d'une vingtaine d'années, la fripe connaît un renouveau remarquable, un essor fulgurant, qui se signale par une multiplication des fripiers et par un engouement des citadins pour les articles fripés, qui ne concernent plus seulement les vêtements mais aussi les chaussures, le linge de maison, les accessoires de mode, etc. Comment expliquer ce nouvel enthousiasme pour des fripes?

Ce changement d'orientation dans les représentations sociales de la fripe nous semble directement lié à la crise économique durable et profonde que vit la ville et aux redéfinitions statutaires consécutives à l'appauvrissement corrélatif des citadins. Mais d'autres facteurs agissent dans le sens de ce changement. Ainsi, la composition des ballots de fripe a évolué. Ceux-ci ne sont plus seulement composés d'articles usagés mais également d'articles neufs, produits de déstockages ou de faillites commerciales qui constituent de «bonnes» occasions. Enfin une conséquence inattendue de la mondialisation a eu pour effet l'arrivée massive de produits asiatiques sur les marchés locaux, dont la qualité est considérée comme étant plus mauvaise que la fripe importée des pays d'Europe occidentale. L'évolution du contexte économique à Kinshasa a ainsi métamorphosé la fripe, ce rebut importé jadis méprisé, en ressource hautement valorisée qui permet aux citadins de rester élégants malgré la crise. C'est ainsi qu'au fil des ans, la friperie est (re)devenue un commerce prospère dont les réseaux de recyclage au détail se sont complexifiés. 
Lors de notre enquête ethnographique chez les fripiers du Grand marché de Kinshasa, nous avons observé que les transactions étaient assez proches de celles observées par Hervé Sciardet aux Puces de Saint-Ouen (2003). Comme à Saint-Ouen, les ballots sont déballés à l'aube et les transactions commerciales se déploient dans les trois principales phases que sont le déballage, l'étalage et la vente au réseau personnel. Cependant à Kinshasa, il y a aussi le recyclage commercial qui est un processus complexe comprenant la sélection, la classification et la requalification des objets.

Dans les lignes qui suivent, après avoir décrit comment le contexte de crise à Kinshasa a influé sur la modification des habitudes vestimentaires, nous examinerons en détail le processus de requalification locale des vêtements d'occasion, avant de nous pencher sur l'évolution des représentations sociales de ces vêtements. Nous axerons la discussion finale sur le processus de «fabrication» de la valeur dans le recyclage commercial de la fripe, en nous appuyant sur les travaux de Jean Baudrillard (1972).

\section{La fripe, l'élégance et le contexte de crise}

Les habitants de Kinshasa, comme ceux de Brazzaville, sont connus en Afrique pour leur goût marqué pour l'élégance vestimentaire. Cette propension à l'élégance a été observée depuis l'époque coloniale, mais c'est surtout dans les années 1980 qu'elle a été portée à son paroxysme par les migrants congolais installés à Paris (Bazenguissa \& Mac Gaffey 1995), incarnée par la «Sape», une société d'élégance dont les membres arborent de manière ostentatoire les griffes de vêtements très coûteux produits par les plus grands couturiers de la planète. Les travaux de l'historien Didier Gondola (1999), qui a étudié la vie quotidienne à Brazzaville et à Kinshasa à l'époque coloniale, ont montré que dès les débuts du vingtième siècle, les nouveaux citadins avaient une forte propension pour l'élégance vestimentaire et que, sous l'influence des missionnaires, ils n'ont pas tardé à adopter les vêtements occidentaux. Après s'être contentés des fripes de leurs employeurs européens, ceux qui le pouvaient financièrement ont commencé à s'acheter des vêtements prêt-à-porter qu'ils exhibaient fièrement les dimanches et jours de fête. Le prêt-à-porter est resté pendant longtemps l'idéal vestimentaire, mais, progressivement, les fripes collectées en Europe par les œuvres caritatives trouvèrent leur place dans les habitudes vestimentaires des citadins. C'est plus particulièrement au début des années 1990, qui inaugura une période de crise sans précédent, qu’on observe un véritable engouement des citadins de Kinshasa pour les vêtements fripés, qui permettent actuellement à beaucoup de familles de s'habiller avec des vêtements de qualité à moindre coût.

Dans cette situation de crise économique durable et de non-emploi structurel, les Kinois ont développé des solutions « informelles» originales qui non seulement approvisionnent la ville en denrées alimentaires, mais aussi donnent du travail et un revenu à des milliers d'entre eux (Ayimpam 2014). C'est dans un tel contexte qu'a eu lieu le renouveau de la fripe à Kinshasa. 


\section{La commercialisation des fripes au Grand marché de Kinshasa}

Le commerce de fripes illustre bien la manière dont un circuit marchand se complexifie au fur et à mesure que l'on change de niveau d'activité entre l'importation et la commercialisation finale au détail. Les ballots de fripes sont généralement importés par des commerçants étrangers, qui peuvent être, selon les cas, de nationalité libanaise, indienne ou pakistanaise. Mais il faut préciser que, même si les importateurs étrangers sont les plus nombreux, la filière de la fripe est en réalité investie par une multitude d'intervenants, aux statuts très divers et à la surface financière très inégale. Ce qui structure tout d'abord cette filière est la capacité des opérateurs à pouvoir interagir en différents lieux à la fois, dans les espaces d'exportation comme dans les espaces d'importation. C'est pourquoi les commerçants étrangers (mais aussi les migrants congolais) sont les principaux importateurs dans ce commerce. Ils distribuent leurs ballots à des grossistes ou des semi-grossistes qui sont le plus souvent eux aussi de nationalité étrangère. Durant les années 1990 et 2000, les magasins de ces derniers étaient généralement situés aux environs du Grand marché. Depuis quelques années, ces magasins se sont progressivement déplacés vers Limete, quartier situé le long du Boulevard de l'aéroport à mi-chemin entre l'aéroport international et le centre historique (Grand marché et centre-ville). Ce déplacement géographique de l'activité des grossistes serait lié, selon nos informateurs, à l'augmentation des volumes importés et stockés, la taille des magasins situés près du Grand marché ne permettant pas le stockage de volumes importants de marchandises.

Ces grossistes travaillent toujours avec des intermédiaires-rabatteurs, les bana kwata, qui se placent à la devanture de leurs magasins. Ces derniers fournissent des informations aux clients, les fripiers du Grand marché, sur l'origine, le contenu et la qualité des ballots. Mais ils jouent aussi le rôle de rabatteur en les incitant à acheter chez tel grossiste plutôt que chez tel autre. Dès que des clients (comme les détaillants vendant dans les autres marchés de la ville) ou des acheteurs occasionnels (comme les colporteurs se rendant en milieu rural) s'approchent du magasin, les bana kwata les interpellent et les abordent en leur demandant ce qu'ils cherchent

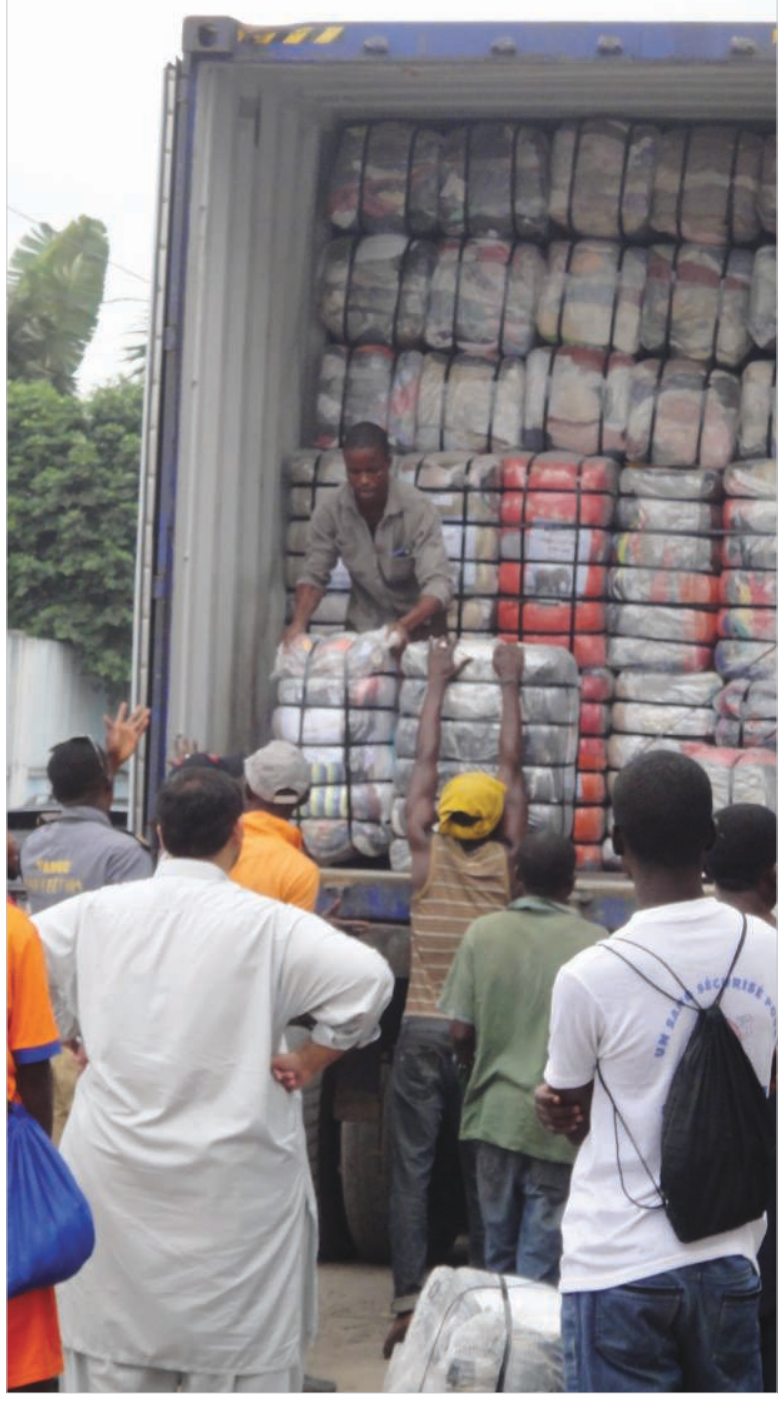

Les ballots de fripe arrivent de l'aéroport. 
2. Le choix du grossiste est important pour la qualité de la fripe et dépend ensuite de la provenance des ballots, et enfin du détail du contenu du ballot. et en vantant la qualité ou le prix des ballots de «leur » grossiste. Mais surtout, avant même que les clients-détaillants ne pénètrent dans la boutique, ils marchandent avec eux pour fixer le prix d'achat des ballots. Ce faisant, ces « rabatteurs» font une marge de profit personnel très confortable, ce qui déplaît fortement à «leur» grossiste qui voit sa propre marge diminuer d'autant.

Aujourd'hui, il n'est plus question pour le fripier de déballer l'ensemble de sa marchandise et de l'étaler à la disposition des clients comme c'était le cas dans le passé. Bien au contraire, l'opération de recyclage, commençant par le déballage aux aurores, est décomposée en plusieurs étapes selon un principe de sélection de la qualité allant de la «fripe chic» à la «fripe cheap». À chaque étape, participent des «trieurs» et des revendeurs qui appartiennent au réseau personnel du fripier revendeur du ballot. C'est le fripier qui choisit qui il convie à chaque niveau de qualité du «tri », les privilégiés étant ceux conviés en premier, à l'aube. Ils peuvent fouiller et dénicher les pièces rares ou les vêtements portant des griffes des grandes marques très prisées à Kinshasa. Ensuite la fripe subit, selon le cas, différentes transformations sur lesquelles nous reviendrons plus loin.

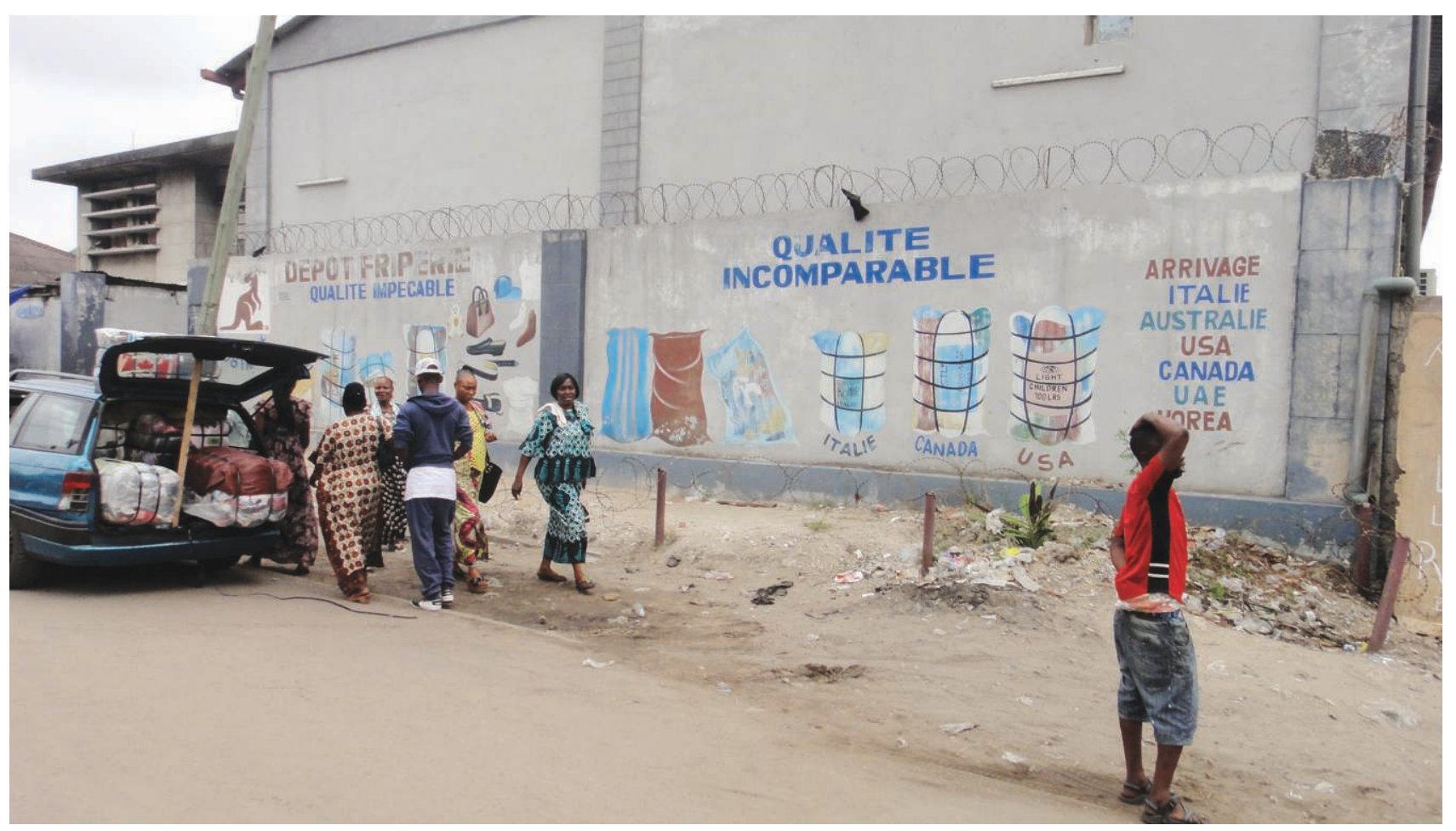




\section{Le recyclage et la requalification de la fripe}

Nous allons décrire ici les différentes étapes de la commercialisation d'un ballot par un commerçant du Grand marché que nous avons suivi, qui est à la fois semi-grossiste et détaillant. Ces étapes sont assez représentatives de la trajectoire des balles de fripes au Grand marché. Vers cinq ou six heures du matin, avant l'ouverture du marché, le commerçant déballe sur son étal au marché. À cette heure, il reçoit des clients particuliers qui sont des «trieurs-revendeurs » qu'il avait informés la veille qu'un nouveau ballot serait déballé le lendemain matin. Ces trieurs sont des intermédiaires qui inspectent le contenu du ballot afin de choisir les meilleures pièces: vêtements de qualité, vestes, chemisiers ou chaussures de grandes marques, draps neufs, serviettes, nappes et couvertures neuves, etc. Le commerçant détaillant fixe alors ses prix. Si le ballot est de très bonne qualité, le commerçant détaillant sait dès cette vente matinale qu'il pourra doubler ou tripler sa mise, alors qu'à cette heure il n'a vendu que le tiers ou le quart du contenu du ballot. Quant aux trieurs-revendeurs, ils jouent le rôle de fournisseur pour les boutiques de luxe ou de prêt-à-porter. Pour réussir dans cette activité, ils doivent connaître les grandes marques, avoir du goût, du flair et un pécule disponible, ce qui n'est pas donné à tout le monde. Mais, avant d'aller revendre ces vêtements aux propriétaires des boutiques

3. Les nouveaux magasins/entrepôts des grossistes sont plus spacieux et permettent plus de stockage de ballots de fripe.

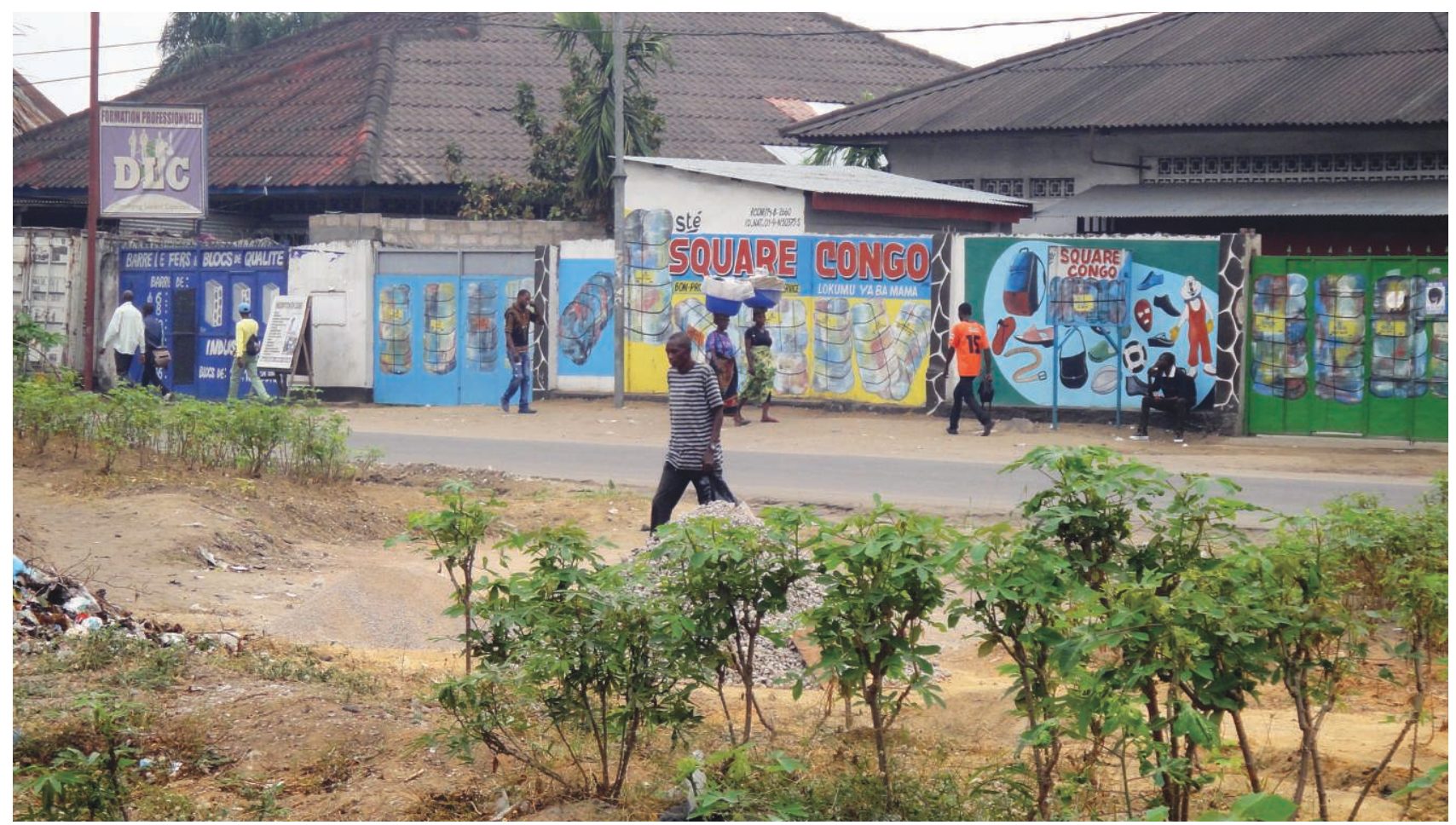


4. Déballage du ballot et premiers trieurs du matin.

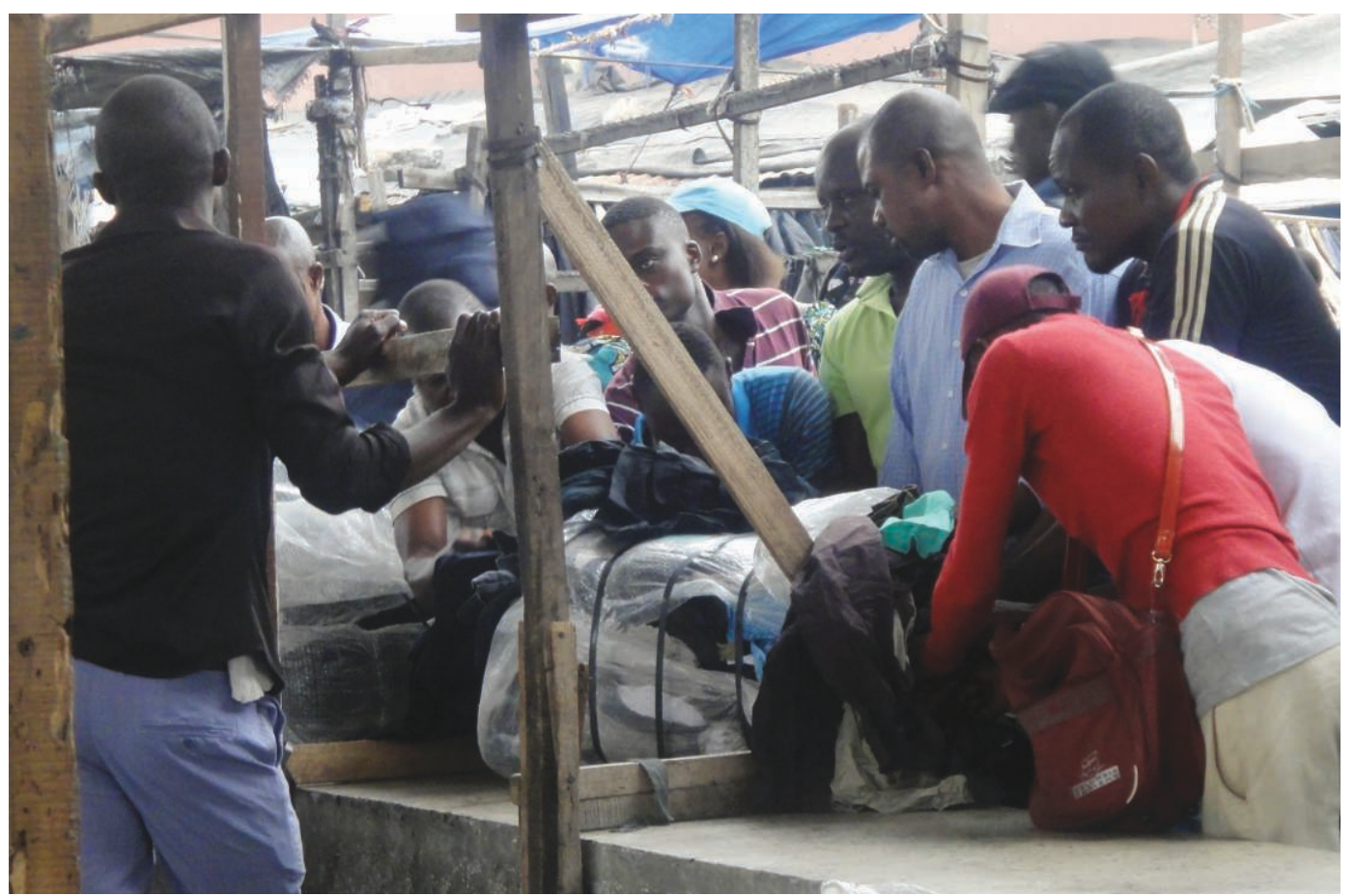

de prêt-à-porter au standing assez élevé, ils s'activent à les faire nettoyer et repasser dans les pressings. Àtitre d'exemple, un matin, pendant le déballage d'un ballot, un trieur-revendeur avait acheté deux costumes quasiment neufs de la marque «Pierre Cardin» à 10 dollars US le costume. Après les avoir emmenés au pressing (où il a payé environ 5 dollars pour chacun), il les a revendus 200 dollars à une boutique chic de Matongé. Le lendemain, les deux costumes étaient en vente en vitrine à 300 dollars le costume.

Après le passage de cette première catégorie de revendeurs, une deuxième catégorie va trier le reste du contenu du ballot pour en extraire les meilleures pièces. Eux sont des commerçants fripiers qui revendent les vêtements soit sur des cintres accrochés sur des fils au long des rues bordant le marché, soit en vente ambulante. Les vêtements sortants des ballots étant chiffonnés et parfois tachés, les vendeurs ambulants doivent les repasser, voire les nettoyer, pour les mettre en valeur et rehausser ainsi leur prix. Pour ce faire, on trouve des petites «buanderies» installées dans des parcelles jouxtant le Grand marché. Là, les revendeurs repassent les vêtements et, dans certains cas, leur font subir quelques modifications afin de les «moderniser» quand les modèles leur semblent un peu vieillots. Les détaillants revendent enfin une partie de leurs ballots à d'autres petits détaillants du marché qui n'ont pas les moyens d'acheter seuls un ballot entier. Étant donné que le prix des ballots, qui varie entre 80 et 300 dollars US, est élevé, il arrive que deux ou trois détaillants s'associent pour acheter ensemble un ballot de fripes. Cette 
pratique permet également de minimiser les pertes, car si le commerce de fripes est très profitable, il comporte aussi beaucoup de risques. Il arrive en effet, qu'un ballot de fripes contienne des vêtements très usés, abîmés, déchirés, tachés, démodés, etc. dont la vente sera difficile ou quasiment impossible. Dans ce cas, le rôle des bana kwata est déterminant, car eux seuls peuvent informer les semi-grossistes détaillants de la mauvaise qualité d'un ballot.

\section{Informel de la fripe "griffée ", informel de la sape}

Il s'est ainsi développé au Grand marché de Kinshasa un «informel» de la fripe «griffée», comme il existe un «informel» de la Sape à Paris et à Bruxelles avec la vente illicite de vêtements «griffés». La recherche effrénée de ceux-ci a produit à Kinshasa tout un trafic de " griffes», entendu ici dans son premier sens d'étiquettes ou de label. Les fripiers qui vendent des vestes sont les plus grands friands et acheteurs de vraies ou fausses « griffes» de grandes marques qui «rehaussent» selon eux la valeur et donc le prix de leurs marchandises. Ces étiquettes griffées peuvent parfois se négocier jusqu'à 50 dollars US la pièce, quand il s'agit des marques les plus prestigieuses de vestes telles que Pierre Cardin, Arthur et Fox ou Yves Saint Laurent. Ce trafic peut aller du négoce de l'étiquette authentique d'une grande marque de veste arrachée à un vieux vêtement, à celui des étiquettes contrefaites. Le prix des étiquettes d'imitation peut lui aussi sensiblement varier selon la dextérité du copieur, allant de l'imitation grossière à une imitation s'approchant de l'originale. Ces griffes sont ostensiblement collées sur le bas des manches ou sur le dos des vestes. Les autres vêtements n'échappent pas à cette forme de contrefaçon.

Mais la contrefaçon dans la fripe ne consiste pas seulement à apposer une marque à un banal vêtement fripé. Elle consiste aussi à faire passer pour de la fripe, des vêtements et chaussures neufs de fabrication chinoise! Ces articles sont généralement vendus à bas prix car ils sont de médiocre qualité. Cette dernière forme de contrefaçon s'adresse à la catégorie des consommateurs qui préfèrent la fripe au prêt-à-porter chinois pour des raisons de qualité. L'opération consiste donc ici à «friper» artificiellement des vêtements neufs, et donc à les froisser, tout en y accolant parfois une marque prestigieuse, ou encore à vieillir artificiellement des chaussures neuves pour les faire passer pour des chaussures usagées, et donc pour des fripes des grandes marques occidentales.

Les sapeurs croient que l'habit fait l'homme et que la griffe fait le vêtement: ils ont de ce fait un rapport quasi fétichiste aux grandes marques (Gondola 1999: 27). Mais les sapeurs parisiens ne semblent pas être les seuls à croire aux griffes et à la différence qu'elles font. Les fripiers du Grand marché à Kinshasa accolent systématiquement des étiquettes de griffes de marques « petites» ou «moyennes» (Zara, Promod, Pimkie, Etam, etc.) à des vêtements qu'ils remodèlent et/ou repassent pour améliorer leur présentation et rehausser leur prix. Selon eux, leurs clients semblent avoir une préférence apriori pour des vêtements qui portent des étiquettes. 


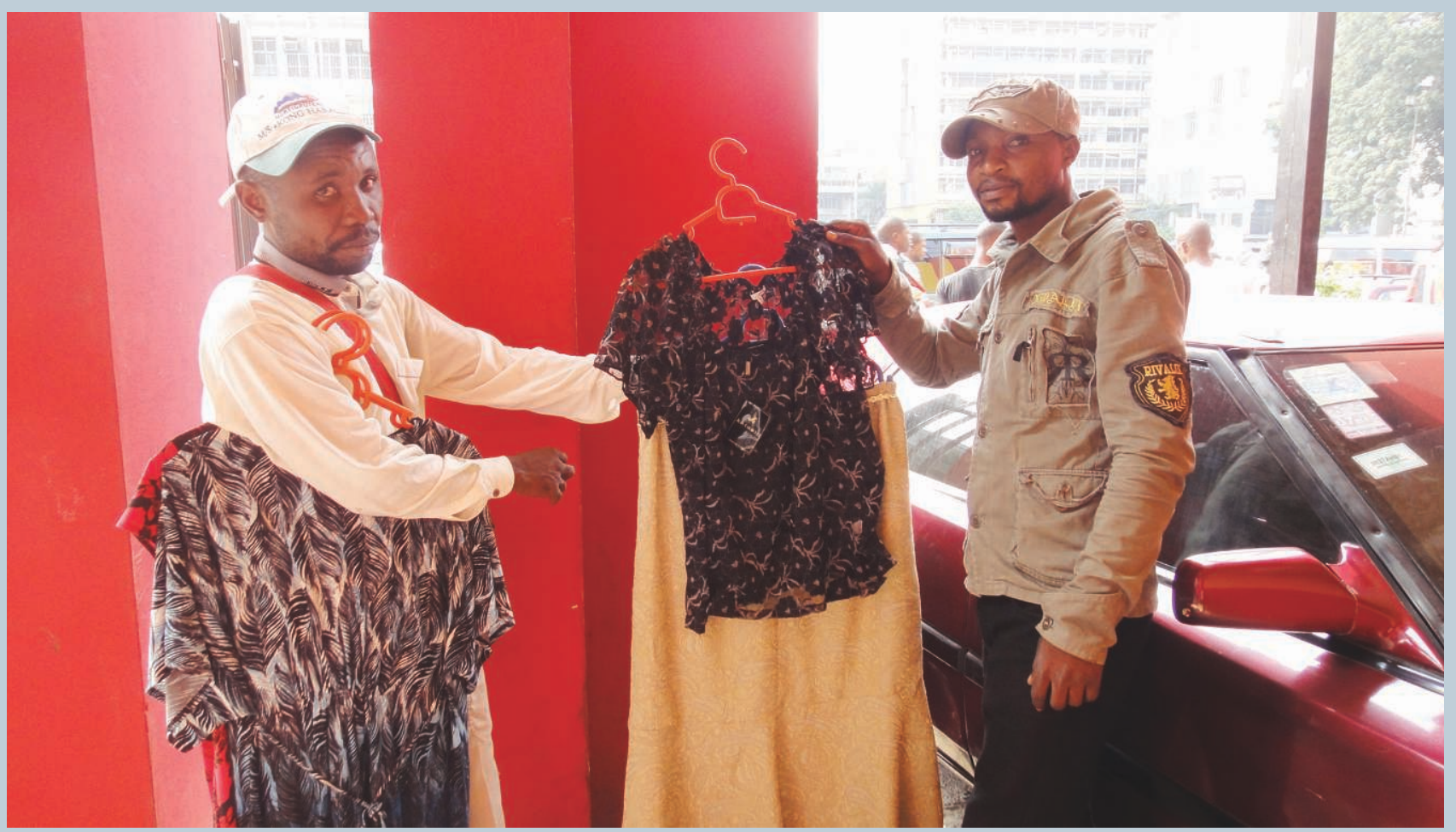

5. La vente ambulante de fripe revalorisée et regriffée. 


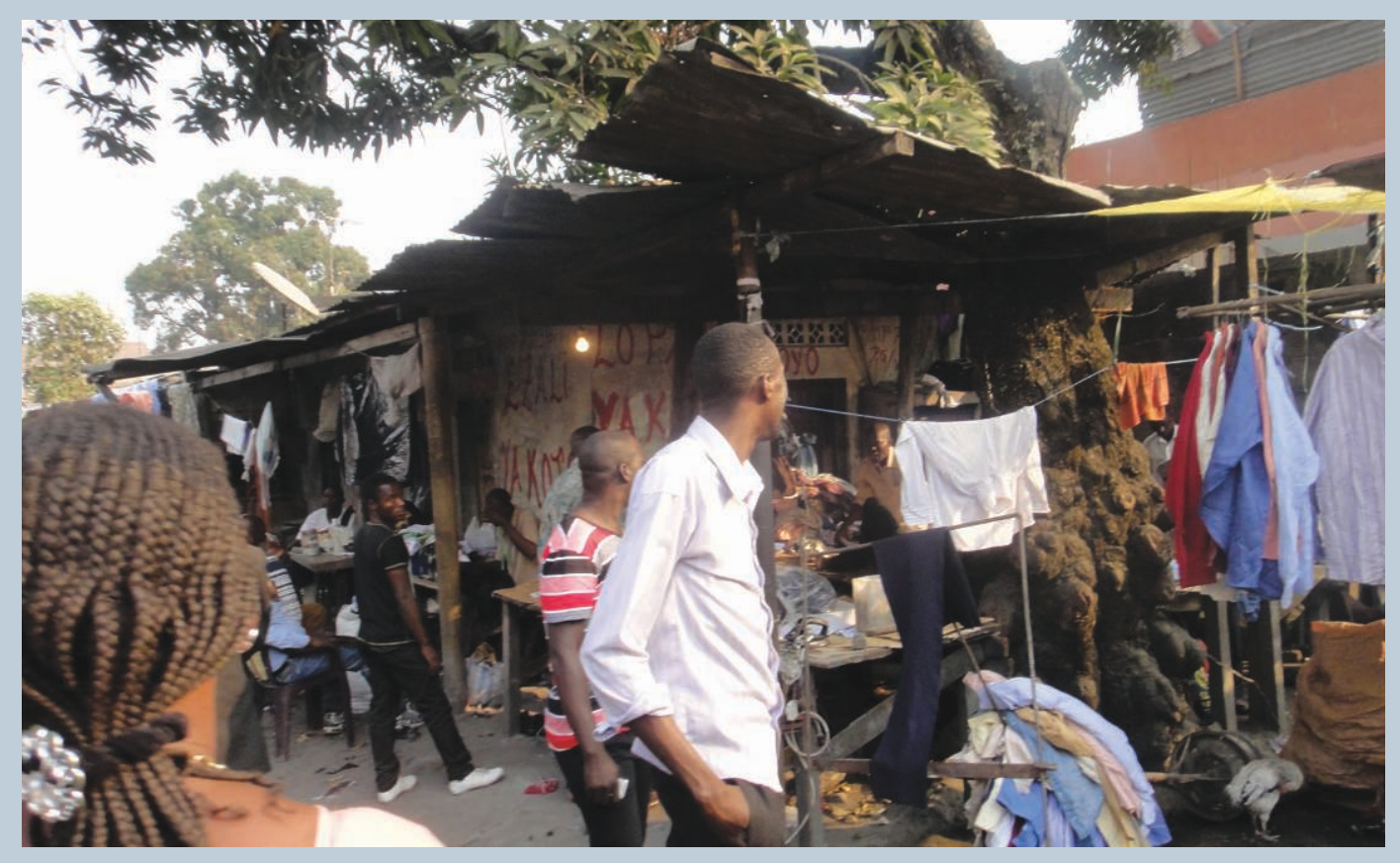

6. Une buanderie populaire, lieu de repassage et de revalorisation de la fripe avant la vente.

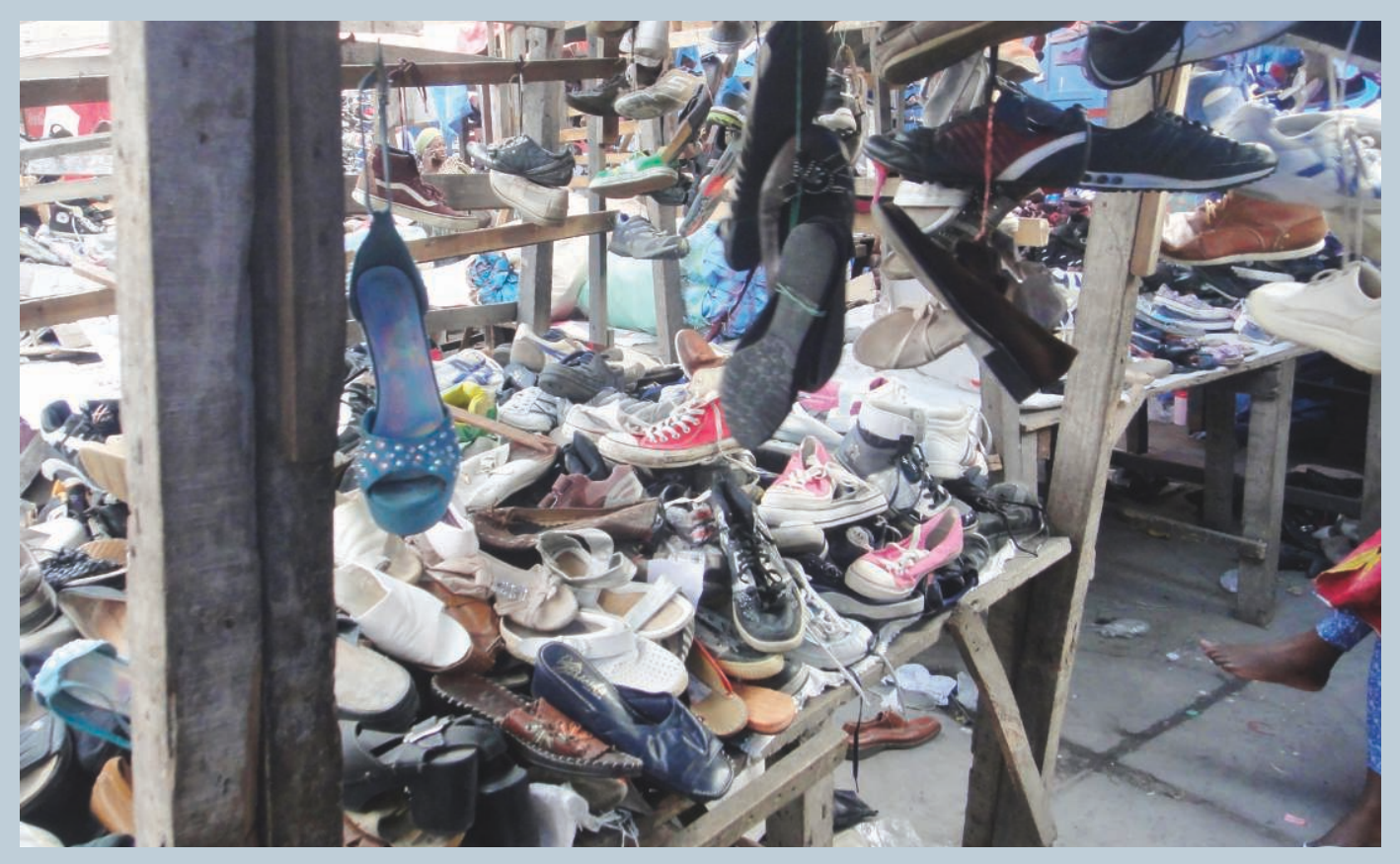

7. Chaussures asiatiques mais artificiellement fripées pour être vendues. 


\section{Le renouveau de la fripe}

La mort annoncée de la fripe en Afrique face à l'arrivée massive des produits chinois à bas prix mais de faible qualité (Bredeloup \& Lombard 2008) ne semble pas avoir touché la ville de Kinshasa. On observe au contraire un renouveau de la fripe, visible à travers une forte expansion de son commerce.

Lors de l'enquête ethnographique menée en 2004-2005, nous avions observé une certaine «migration» de la population marchande qui quittait la place aménagée des pavillons du Grand marché pour s'installer dans les marchés de rue. Cette micro-migration s'accompagnait cependant du «repeuplement» des pavillons ainsi délaissés par d'autres marchands, notamment par les fripiers. Ce «repeuplement» des pavillons a été confirmé lors de l'enquête de 2010. Quant à l'enquête de 2014, elle a montré l'ampleur de l'extension prise par l'activité de la fripe sur la place du marché.

Il est intéressant d'observer par exemple que les pavillons 6 et 7, originellement dédiés à la vente de la viande et de poisson frais, ont été progressivement repeuplés par des tailleurs faisant du raccommodage et de la couture rapide, ainsi que les «tailleurs-remodeleurs» des articles sortis des ballots de fripes, les «buanderies» populaires situées dans les parcelles près du Grand marché ne suffisant plus à faire face à la demande. Sur les étals métalliques où l'on vendait de la viande trônent aujourd'hui des machines à coudre, des rubans, des fils, etc. Ces tailleurs cohabitent avec les derniers marchands de vivres frais. Il en est de même du pavillon 8 qui était dédié auparavant à la vente des fruits et légumes, et qui est actuellement occupé au 9/10 par les marchands de fripe, et par les tailleurs-remodeleurs.

Le plus frappant dans cette redéfinition des espaces à l'intérieur du Grand marché, ce sont les surfaces considérables qu'occupe désormais le commerce de la fripe. Quand on arrive au Grand marché par le sud, par l'ouest, voire par l'est, on a l'impression qu'on arrive dans un énorme marché de fripe; cela donne la fausse impression que c'est le seul produit qui y est vendu.

Il apparaît que les représentations sociales de la fripe à Kinshasa ont évolué; de vêtement d'occasion jadis méprisé et considéré comme un marqueur de pauvreté, elle est aujourd'hui valorisée. La crise, qui a contraint les citadins appauvris à modifier leurs habitudes de consommation, a joué un rôle déterminant dans l'évolution des représentations du vêtement d'occasion. Par ailleurs, la fripe n'est plus seulement vue comme un rebut importé car la composition des ballots de fripes a elle aussi changé. On peut actuellement y trouver des articles neufs, des produits de déstockages ou de faillites commerciales qui constituent pour beaucoup de citadins de «bonnes» occasions. Cependant, si les représentations collectives ont évolué, il faut indiquer que le rapport des citadins à la fripe, demeure ambivalent.

Bien qu'il y ait un indéniable engouement pour la fripe, on observe encore, parfois, la persistance d'un jugement méprisant, décrivant un vêtement d'occasion, de seconde main, usagé, dégradé, souillé et destiné aux «pauvres». Se perpétue aussi chez certains citadins la représentation de la fripe comme vecteur de transmission des maladies en provenance des pays occidentaux, et notamment la tuberculose et le Sida, présentant un danger pour la santé de 


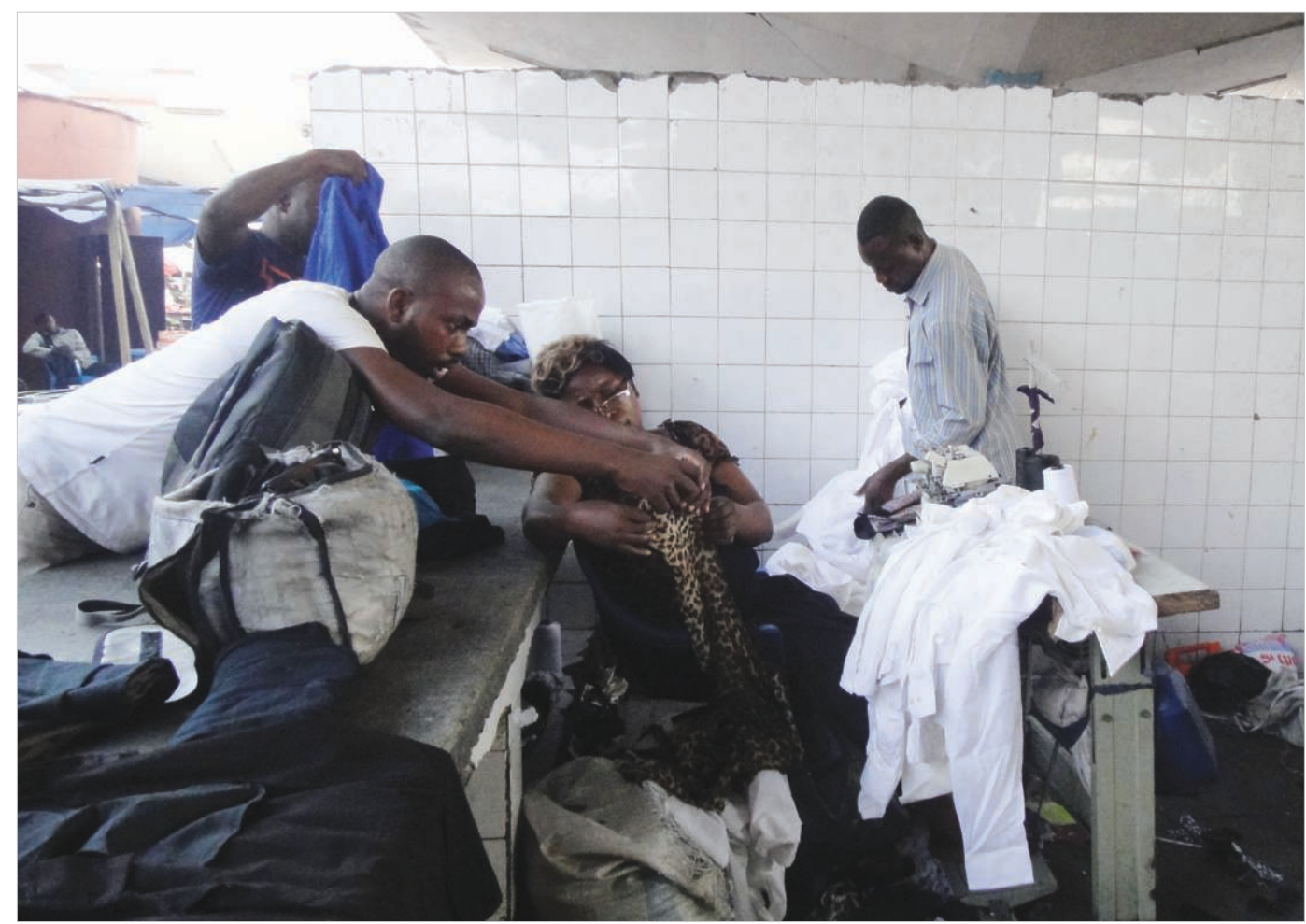

ceux qui la portent; ces mêmes pays occidentaux qui déverseraient sur le africain leurs rebuts, comme la fripe, et leurs déchets « poubelles» transformant les villes africaines qui les reçoivent en espaces « dépotoirs».

\section{Au-delà de la valeur d'usage : une "fabrication " différentielle de la valeur}

La discussion finale de cette contribution portera sur le processus de «fabrication» de la valeur dans le recyclage commercial de la fripe à Kinshasa. On s'appuiera pour ce faire sur les travaux de Jean Baudrillard (1972) qui a repris la discussion classique sur la dichotomie valeur d'usage/ valeur d'échange. La thèse de Jean Baudrillard est particulièrement éclairante pour clarifier le statut ambigu de la fripe en tant que rebut qui se métamorphose en marchandise valorisée. Comme le note Baudrillard (1972: 154), on sait que la marchandise est à la fois valeur d'usage et valeur d'échange. Reprenant Marx (Le capital, livre I, VI), il avance que pour devenir une marchandise (le support d'une valeur d'échange) l'objet -ici le rebut - doit satisfaire un quelconque 
besoin social en ayant la propriété utile correspondant. La valeur qui est à l'origine du besoin social est elle aussi socialement construite. La fabrication de la valeur différentielle des objets fripés par les trieurs-revendeurs s'appuie sur une évaluation des fripes sortant des ballots qui met en correspondance les propriétés utiles des fripes en vrac avec les codes particuliers aux besoins sociaux locaux en habillement. Cette évaluation se fonde sur la logique fonctionnelle de la valeur d'usage potentielle des vêtements fripés.

La première catégorie concerne la modalité de recyclage mise en place par les «trieurs de l'aube», les privilégiés, ceux-là même qui sont invités aux premières heures du matin à trier dans le ballot juste au déballage pour « dénicher» les pièces rares, les vêtements griffés. Pour cette catégorie, la valeur, le signifié de distinction, est déjà contenu dans l'objet lui-même qui est reconnu comme tel. Sa valeur d'échange finale sera en correspondance avec cette valeur de distinction. La deuxième modalité concerne les trieurs qui font subir aux vêtements un remodelage, c'est-à-dire des transformations de modèles un peu vieillots, ou un peu abîmés. Ces derniers vont reconstruire la valeur d'usage du vêtement fripé qui est en l'état comme une matière première ne présentant a priori aucun signe valorisant. Ils mettent alors en œuvre un processus de valorisation qui va transformer le rebut en marchandise, soit en transformant les

9. Tailleurs-remodeleurs occupant le pavillon 8.

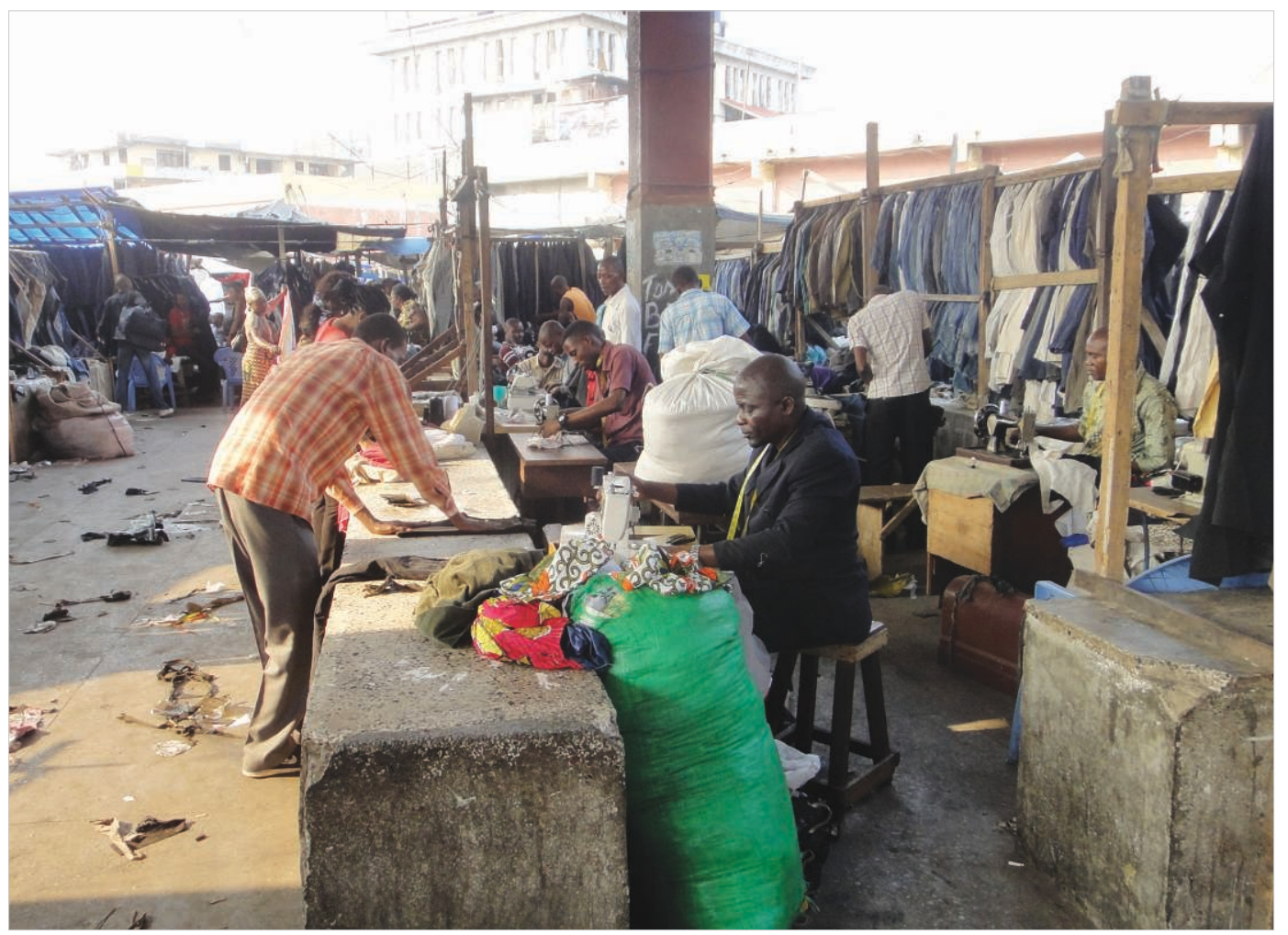


modèles d'origine ou en taillant d'autres modèles «à la mode» à partir de ces vêtements. Dans cette modalité de fabrication de la valeur, il y a une variante, dans laquelle les trieurs procèdent non pas au remodelage des fripes, mais à leur revalorisation simple. L'objet lui-même présente des signes négatifs: froissures, déchirures, etc., qui altèrent la valeur symbolique de l'objet et, par conséquent, sa future valeur marchande.

Le processus de repassage, de raccommodage auquel se livrent les trieurs dans les buanderies populaires revalorise l'objet en améliorant sa présentation (au terme du processus, il sera présenté de manière impeccable sur des cintres), l'objectif recherché de cet encodage positif étant d'accroître la valeur marchande finale de l'objet.

Les «trieurs de l'aube» fabriquent la valeur symbolique en «travaillant» des objets qui présentent d'emblée, ou auxquels ils apposent a posteriori, les signes de la valeur recherchée par une clientèle en quête de vêtements « griffés»: les adeptes locaux de la sape qui fonctionnent selon un code de distinction que les trieurs connaissent parfaitement. Même quand ils travaillent sur des vêtements réellement griffés, ils usent de procédés qui consistent à faire passer pour neufs des vêtements usagés en les faisant nettoyer dans les pressings avant de les présenter aux boutiques de luxe. Il s'agit là d'un véritable travail de mise en scène de la valeur différentielle.

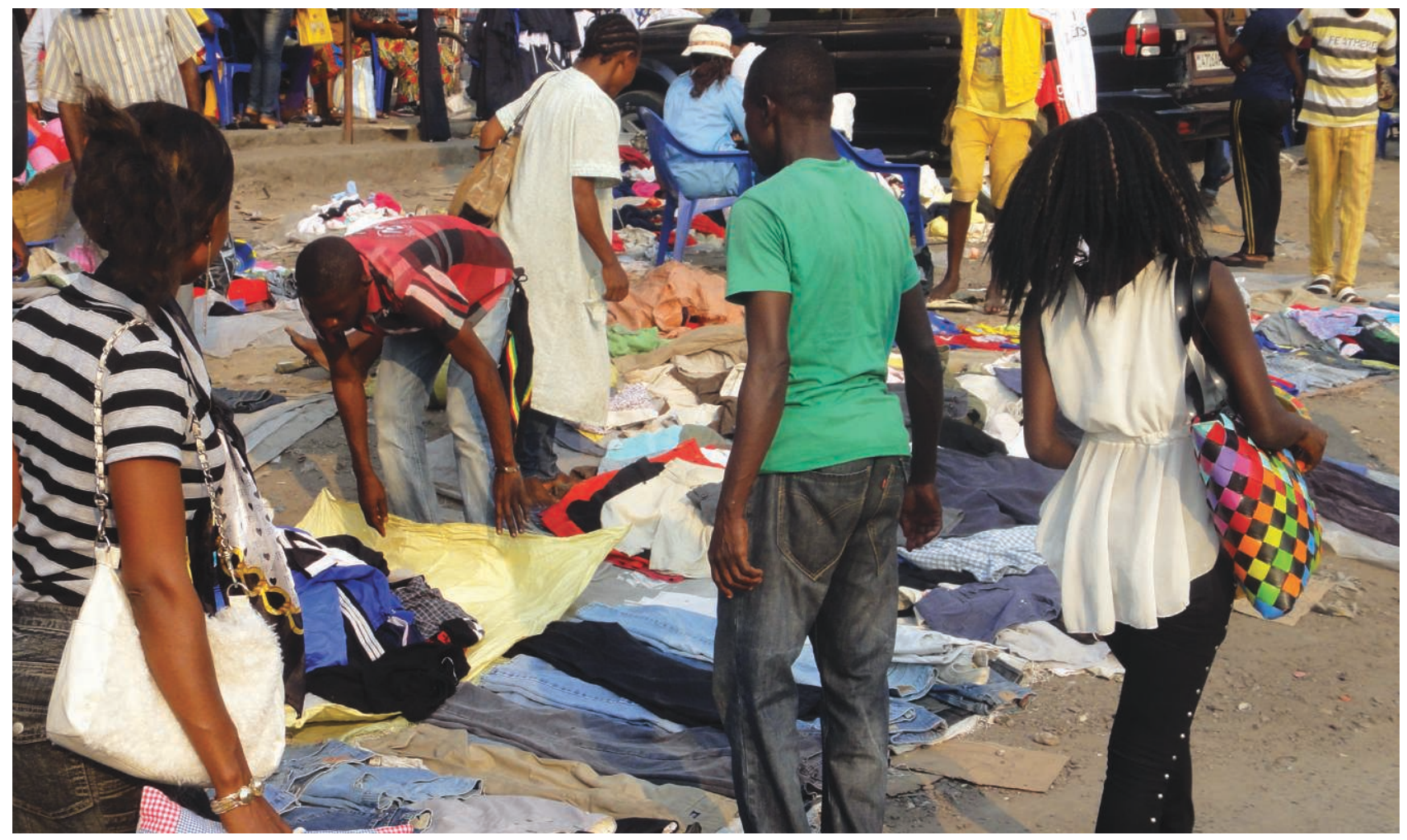


Ils fabriquent de la valeur symbolique matériellement en reconditionnant, en jouant sur les représentations collectives des signes valorisés dans leur société.

Il faut dire que la sape est une représentation de la valeur des fringues: c'est là où la fripe rencontre la sape. Les gens y mettent le prix parce que ce créneau est reconnu comme celui de la valeur de distinction finale. La sape existe comme système de réponse collective de la valeur de distinction reconnue par tous. Les fripiers le savent, les trieurs-revendeurs également. Et ils arrivent à produire des objets valorisés par les sapeurs, soit en «trouvant » les objets authentiques dans les ballots, soit par des contrefaçons. C'est un marché qui fonctionne exclusivement sur la représentation symbolique de la valeur différentielle du signe. Quant aux non-sapeurs, ils misent sur la valeur d'usage des objets qui se présentent comme étant propres, bien présentés, refaçonnés, «refashionnés » correspondant aux valeurs symboliques de la mode ambiante: c'est le segment dans lequel Monsieur et Madame «tout le monde» ont l'air élégant, car l'élégance vestimentaire est une valeur cardinale de la société urbaine kinoise.

On a tenté dans cette contribution d'interroger les pratiques de recyclage de la fripe dans un contexte de crise économique comme celui de la ville de Kinshasa, en se demandant ce que ce rebut importé mais localement recyclé et revalorisé pouvait révéler des ressorts économiques, sociaux et surtout symboliques de la relation des citadins aux vêtements d'occasion. Nous avons vu que la situation socio-économique a poussé nombre de citadins à changer leurs comportements de consommation du vêtement et d'autres à s'investir massivement dans des activités de recyclage commercial de la fripe pour en faire leur gagne-pain. Nos observations montrent que si ce contexte de crise a indéniablement influencé l'évolution des représentations sociales du vêtement d'occasion, il ne s'agit pas pour autant du désir d'accéder à un mode de vie plus durable. Le renouveau de la fripe à Kinshasa semble se situer à notre sens entre la nécessité pour les citadins de continuer à s'habiller et à rester élégants malgré la disqualification sociale relative à la crise, et celle de trouver des moyens de survivre. Le processus de recyclage commercial de la fripe est pour beaucoup dans ce renouveau. Nettoyée au pressing ou dans les buanderies populaires, repassée, réparée, ré-étiquetée, remodelée ou transformée, la fripe, devenue «méconnaissable», peut se retrouver aussi bien dans les boutiques de luxe que chez les revendeurs ambulants, au cintre, étalée ou en vrac au marché. C'est cette «fabrication» différentielle d'une valeur symbolique localement ajoutée dans le processus du recyclage local qui métamorphose le rebut (la fripe) en marchandise (vêtement valorisé et valorisant). 


\section{Remerciements}

Je voudrais remercier Yorick Biembe dont l'aide m’a été précieuse durant l'enquête de juin et de juillet 2014, ainsi que Jacky Bouju pour ses critiques et ses commentaires sur une précédente version de ce texte.

\section{I'auteure}

Sylvie Ayimpam est socio-anthropologue et docteure en sciences politiques et sociales de l'université catholique de Louvain. Elle est chercheure associée à l'Institut des mondes africains (IMAF) à Aix-en-Provence. Elle est actuellement Team leader pour le Congo-Kinshasa dans le cadre du programme «Ethnographic Study on Mobile Money in Africa» de l'African Studies Centre Leiden (Pays-Bas). Ses recherches portent notamment sur les stratégies de survie en milieu populaire urbain, les violences sociales ordinaires, la régulation sociale en contexte de crise, l'informalité en contexte migratoire. Elle a récemment publié Économie de la débrouille à Kinshasa. Informalité, commerce et réseaux sociaux (Karthala, 2014), et a codirigé avec Jacky Bouju Enquêter en terrains difficiles. Objets tabous, lieux dangereux, sujets sensibles (Civilisations 64, 2015).

\section{Iconographie}

Toutes les images (dont image d'ouverture). (C) S. Ayimpam.

\section{Références}

Ayimpam, S. 2014 Économie de la débrouille à Kinshasa. Informalité, commerce et réseaux sociaux. Paris: Karthala.

Baudrillard, J. 1972 Pour une critique de l'économie politique du signe. Paris: Gallimard.

Bazenguissa, R. \& J. Mac Gaffey 1995 «Vivre et briller à Paris. Des Jeunes Congolais et Zaïrois en marge de la légalité économique», Politique africaine 57: 124-133.

Bordreuil, J.S. 1981 « Notes pour une sociologie de la valeur d'usage», L'Homme et la société 59-62 Imaginaire social et créativité: 55-73.
Bredeloup, S. \& J. Lombard 2008 «Mort de la fripe en Afrique ou fin d'un cycle?», Revue Tiers-Monde 194 (2): 391-412.

Gondola, D. 1999 «La sape des mikilistes. Théâtre de l'artifice et représentation onirique », Cahiers d'études africaines 39 (153): 13-47.

Sciardet, H. 2003 Les marchands de l'aube. Ethnographie et théorie du commerce aux Puces de Saint-Ouen. Paris: Economica.

\section{Pour citer cet article}

Ayimpam, S. 2016, «La valorisation du rebut. Le recyclage commercial de la fripe en contexte de crise», Techniques ECulture 65-66 «Réparer le monde. Excès, reste et innovation», p. 264-279. 